\title{
Overexpression of Brassica rapa GROWTH-REGULATING FACTOR genes in Arabidopsis thaliana increases organ growth by enhancing cell proliferation
}

\author{
Joon Ki Hong $\cdot$ Seon-Woo Oh $\cdot$ Jeong Hoe Kim $\cdot$ Seung Bum Lee $\cdot$ Eun Jung Suh $\cdot$ Yeon-Hee Lee
}

Received: 6 July 2017 / Revised: 28 August 2017 / Accepted: 19 September 2017

(c) Korean Society for Plant Biotechnology

\begin{abstract}
GROWTH-REGULATINGFACTOR $(G R F)$ genes encode plant-specific transcription factors containing two conserved QLQ and WRC domains and play critical roles in regulating the growth and development of lateral organs, such as cotyledons, leaves, and flowers. To explore the agricultural potential of Brassica rapa $G R F$ genes $(B r G R F \mathrm{~s})$, the researchers isolated seven $B r G R F s$ (BrGRF3-1, 3-2, 5, 7, 8-1, 8-2, and 9) and constructed $B r G R F$-overexpressing Arabidopsis thaliana plants (BrGRF-OX). BrGRF-OX plants developed larger cotyledons, leaves, and flowers as well as longer roots than the wild type. The increase in size of these organs were due to increases in cell number, but not due to cell size. $B r G R F-O X$ plants also had larger siliques and seeds. Furthermore, $B r G R F$-OX seeds produced more oil than the wild type. RT-PCR analysis revealed that $B r G R F \mathrm{~s}$ regulated expression of a wide range of genes that are involved in gibberellin-, auxin-, cell division-related growth processes. Taken together, the data indicates that $B r G R F$ s act as positive regulators of plant growth, thus raising the possibility that they may serve as a useful genetic source for crop improvement with respect to organ size and seed oil production.
\end{abstract}

Keywords Cell division, GROWIH-REGULATINGFACTOR, Morphogenesis, Organ size, Seed oil, Transcription factor

\footnotetext{
J. K. Hong · S.-W. Oh · S. B. Lee · E. J. Suh · Y.-H. Lee $(\varangle)$ National Institute of Agricultural Science, Rural Development Administration, 370 Nongsaengmyeong-ro, Jeonju-si, Jeollabuk-do, Korea

e-mail: yhl2222@korea.kr

J. H. Kim

Department of Biology, Kyungpook National University, 1370

Sankyuk-dong, Buk-gu, Daegu 702-701, Korea
}

\section{Introduction}

GROWTH-REGULATING FACTOR genes (GRFs) encode plant-specific transcription factors, which play a critical role in regulation of plant growth and development (Kim and Tsukaya 2015; Omidbakhshfard et al. 2015). The first $G R F$ gene was identified in rice, being suggested to perform a regulatory role in stem elongation induced by the phytohormone gibberellin (Van der Knaap et al. 2000). GRF proteins contain two highly conserved QLQ (Gln, Leu, Gln) and WRC (Trp, Arg, Cys) domains in the N-terminal region. The QLQ domain serves as an interface for interaction with GRF-INTERACATING FACTORs, which act as transcription co-activators (Horiguchi et al. 2005; Kim and Kende 2004). The WRC domain has two distinctive structural features, namely a putative nuclear localization signal (NLS) and a zinc-finger motif $\left(\mathrm{C}_{3} \mathrm{H}\right.$ motif) for DNA binding (Van der Knaap et al. 2000). The C-terminal region of GRFs is involved in the transactivation activity.

$G R F s$ have been isolated and characterized in a wide range of plant species, including Arabidopsis thaliana, Brassica napus, Brassica rapa, Glycine max (soybean), Solanum tuberosum (potato), Oryza sativa (rice), Zea mays (maize), Physcomitrella patens (moss), Marchantia polymorpha (liverwort), and other land plants (Kim and Tsukaya 2015; Omidbakhshfard et al. 2015). Many studies have reported that $G R F \mathrm{~s}$ act as positive regulators of cell proliferation and thus determine the final size of lateral organs, such as cotyledons, leaves, and flowers (Horiguchi et al. 2005; Kim and Kende 2004; Kim et al. 2003; Liu et al. 2009; Wang et al. 2014). GRFs also play a role in development of female reproductive organs of $A$. thaliana (Liang et al. 2014), and are expressed in rice embryos and maize ears, suggesting a possible role in regulation of seed growth and development (Ye et al. 2004; Zhang et al. 2008). A GRF gene of B. napus, $B n G R F 2$, was proposed to be responsible for increases in 
seed weight, probably by regulating cell number and photosynthesis; these, in turn, might be also responsible for the increased production of seed oil (Liu et al. 2012).

In the complete genome sequence of $B$. rapa, 17 nonredundant $G R F \mathrm{~s}(B r G R F \mathrm{~s})$ have been identified (Wang et al. 2014). BrGRF proteins are more closely related to those of $A$. thaliana (AtGRFs) than those of $O$. sativa (OsGRFs). $B r G R F$ s were expressed in specific tissues and organs, and the transcription of most $B r G R F \mathrm{~s}$ was induced by the phytohormone gibberellic acid (GA). The overexpression of $B r G R F 8$ in A. thaliana plants increased the size of leaves and other organs by regulating cell proliferation rather than cell volume, suggesting that $B r G R F \mathrm{~s}$ might also control organ growth and development by regulating cell proliferation, as did some of $A t G R F$ s.

In this study, the function of $B r G R F \mathrm{~s}$ isolated from $B$. rapa L. (ssp. pekinensis cv. JangWon) were investigated using overexpression strategies. As a result, we demonstrate that $B r G R F$ s play an important role in regulation of organ growth and propose that these genes could serve as a useful tool for genetic manipulation leading to improvement of agronomical traits of crops.

\section{Material and Methods}

Plant material and growth conditions

Brassica rapa L. (ssp. pekinensis cv. JangWon) seeds were sown on MS medium (Murashige and Skoog 1962) containing $3 \%$ sucrose and $0.25 \%$ phytagel $(\mathrm{pH} 5.8)$ and stratified at $4^{\circ} \mathrm{C}$ for 5 days in darkness to induce synchronous germination. The plants were grown at $23^{\circ} \mathrm{C}$ for 2 weeks under long-day conditions (16-h light and 8-h dark), after which they were transplanted to soil and transferred to a growth chamber for further culture until use.

Arabidopsis thaliana Columbia seeds (Col-0) were surfacesterilized by vapor-phase sterilization (http://www.plantpath. wisc.edu/fac/afb/vapster.html; Desfeux et al. 2000) and plated on MS medium supplemented with vitamins, $1 \%$ sucrose, and $0.3 \%$ phytagel (Sigma, St. Louis, MO, USA). The seeds were stratified at $4^{\circ} \mathrm{C}$ for 2 days in the dark and then transferred to a growth chamber under a 16-h light/8-h dark photoperiod at $23^{\circ} \mathrm{C}$.

Isolation of $B r G R F \mathrm{cDNAs}$ and construction of expression vectors

$B r G R F$ cDNAs were synthesized using RNA extracted from shoot apical tissues of 3- to 4-week-old B. rapa plants. Three micrograms of total RNA were reverse-transcribed in a $30-\mu 1$ reaction volume with $1 \mu \mathrm{g}$ of oligo $(\mathrm{dT})_{18}$ primer using MMLV reverse transcriptase (RNaseH free), according to the manufacturer's instructions (TOYOBO, Osaka, Japan). One microliter of the cDNA solution after dilution (1:3) was used for PCR amplification $\left(95^{\circ} \mathrm{C}\right.$ for $30 \mathrm{sec}, 55^{\circ} \mathrm{C}$ for $30 \mathrm{sec}, 72^{\circ} \mathrm{C}$ for $45 \mathrm{sec}$ ) in a volume of $25 \mu \mathrm{l}$ with gene-specific primer sets (Supplemental Table 1). All PCR products of accurate sizes were cloned into the pGEM-TEasy vector (Promega, Madison, WI, USA), and confirmed by DNA sequencing.

In order to construct plant expression vectors, each $B r G R F$ cDNA fragment was inserted into the sense orientation between the cauliflower mosaic virus (CaMV) $35 S$ promoter (35S-pro) and the nopaline synthase terminator (nos-ter) of pCAMBIA with $h p t$ as a selectable marker gene. BrGRF5 cDNA was inserted into the sense orientation between the 35S-pro and $35 S$ polyA (T35S) of pB2GW7 with bar as a selectable marker gene (Supplemental Fig. 1A).

Generation and verification of transgenic plants

Flowers of A. thalina were sprayed with Agrobacterium tumefaciens GV3101 cells with the recombinant binary vectors suspended in 5\% sucrose and $0.05 \%$ Silwet-L77. The plants were then incubated in a growth chamber at $23^{\circ} \mathrm{C}$ and $100 \%$ humidity for 1 day, and allowed to grow in a growth chamber under a 16-h light/8-h dark photoperiod at $23^{\circ} \mathrm{C} . \mathrm{T}_{1}$ seeds and their progeny seeds were allowed to germinate on MS medium containing $50 \mathrm{mg} / \mathrm{L}$ hygromycin or $25 \mathrm{mg} / \mathrm{L}$ phosphinothricin for selection of transgenic plants. Transgenic plants with a single T-DNA insertion and a high level of $B r G R F$ expression were chosen for further experimentation and were grown at $23^{\circ} \mathrm{C}$ during day and $21^{\circ} \mathrm{C}$ during night under a long-day photoperiod (16-h light). Phenotypes of transgenic plants were characterized at the developmental stages indicated.

To confirm the presence of transgenes in the selected plants, we performed PCR using genomic DNA with hpt-, bar-, and $B r G R F$-specific primer sets (Supplemental Table 1). PCR reactions were performed using 25 cycles of 30 sec at $95^{\circ} \mathrm{C}, 30 \mathrm{sec}$ at $55^{\circ} \mathrm{C}$, and $45 \mathrm{sec}$ at $72^{\circ} \mathrm{C}$, followed by a final extension at $72^{\circ} \mathrm{C}$ for $10 \mathrm{~min}$.

For quantitative RT-PCR analysis of $B r G R F$ transgenes, total RNA was extracted from leaves. Two micrograms of total RNA were converted to cDNA. One microliter of the cDNA solution after dilution (1:3) was used for PCR amplification with $B r G R F$-specific primer sets. The expression level of Actin (At3g18780) was used as a quantitative control. 
Phenotypic characterization of transgenic plants

Digital images of detached leaves, cotyledons, and petals were acquired using a scanner, and their dimensional parameters (area, length, and width) were determined with the image-analyzing program SCIONIMAGE (Scion, Frederick, MD, USA).

In order to analyze cell number and size, plant tissues were cleared in Visikol VSKP-100 for 1 day. All microscopic images were obtained using an Axio imager M1 microscope installed with Axiovision4 microscopy software (Carl Zeiss Jena GmbH, Germany). The numbers of palisade cells in the subepidermal layer aligned along a longitudinal axis just beside the midvein or along a transverse axis in the maximum width region were counted. To determine cell area, 20 cells grouped halfway from the midvein to the leaf margin at the widest point were analyzed with SCIONIMAGE. Each sample was replicated three times. Areas of organs and cells were subsequently used to calculate total numbers of cells.

Measurement of lipid production in seeds

Total contents of seed lipids represent the sum values of 15 different fatty acids identified by gas chromatography (GC) analysis: decanoate (10:0), laurate (12:0), tridecanoate (13:0), miristate (14:0), palmitate (16:0), palmitoleate (16:01), heptadecanoate (17:0), stearate (18:0), oleate (18:1), linoleate (18:2), linolenate (18:3), arachidate (20:0), eicosenoate (20:1), behenate (22:0), and erucate (22:1). Individual fatty acids were determined according to the AOCS method Ce 1-62 (AOCS 1997) by using a GC 7890B (Agilent Technology, America). Transgenic $A$. thaliana seeds were crushed with chrome beads ( $2.3 \mathrm{~mm}$ dia. Cat No. $11079123 \mathrm{c}$ ) by Tissue
Analyser II (Qiagen, Japan). Fatty acids were extracted using the $\mathrm{CHCl}_{3}: \mathrm{MeOH}(2: 1)$ solution. The pentadecanoic acid solution (1000 ppm) was used as an internal standard. Quantities of individual fatty acids were calculated from their chromatographic peaks based on a regression equation of the internal standard.

Expression profiles of growth- related genes

To analyze the transcript levels of cell division-, GA-, and auxin-related genes, total RNA was extracted from shoot apical tissues and leaves using a Trizol reagent (Invitrogen, Carlsbad, CA, USA). We performed semi-quantitative RTPCR using primer sets corresponding to following genes: 15 GA-related (Supplemental Table 2, Fleet and Sun 2005), 12 auxin-related (Supplemental Table 3, Li et al. 2007), and 39 cell division-related (Supplemental Table 4, Gonzalez et al. 2009; Krizek 2009). Relative expression levels were determined by semi-quantitative RT-PCR amplification using Quantity One (Bio-Rad, Hercules, CA, USA; Liang et al. 2011) and (http://biochemlabsolutions.com/GelQuantNET.html).

\section{Results and Discussion}

Establishment of $B r G R F$-overexpressing A. thaliana plants

As mentioned above, an earlier study identified and characterized all 17 non-redundant BrGRFs (Wang et al. 2014). To investigate their biological function in detail, we isolated $10 B r G R F$ cDNAs and renamed them BrGRF1, 2-1, 2-2, 3-1, $3-2,5,7,8-1,8-2$, and 9 on the basis of the similarity to the AtGRF orthologues according to the phylogenetic relationship (Table 1, Supplemental Fig. 2). Comparison of

Table $1 A t G R F$ family members and accession numbers of BAC clones from the B. rapa genome database

\begin{tabular}{|c|c|c|c|c|c|c|c|}
\hline $\begin{array}{l}\text { Arabidopsis } \\
\text { gene name }\end{array}$ & AGI & BAC clone ${ }^{\mathrm{a}}$ & $\begin{array}{c}\text { BAC } \\
\text { Acc. No. }\end{array}$ & $\begin{array}{c}\text { B. rapa } \\
\text { gene name }\end{array}$ & CDS Acc. No. & CDS (bp) & Amino acid \\
\hline AtGRF1 & At2g22840 & KBrH110M01 & AC237306 & $B r G R F 1$ & JN698686 & 1557 & 518 \\
\hline \multirow{2}{*}{$A t G R F 2$} & \multirow{2}{*}{ At4g37740 } & KBrB006J12 & AC189204 & $B r G R F 2-1$ & JN698687 & 1356 & 451 \\
\hline & & KBrH005P10 & AC189552 & $B r G R F 2-2$ & JN698688 & 1344 & 446 \\
\hline \multirow[t]{2}{*}{$A t G R F 3$} & \multirow[t]{2}{*}{ At2g36400 } & \multirow[t]{2}{*}{ KBrB031G07 } & \multirow[t]{2}{*}{ AC189304 } & $B r G R F 3-1$ & $\mathrm{Bra} 023066$ & 1239 & 368 \\
\hline & & & & $B r G R F 3-2$ & Bra005268 & 1167 & 388 \\
\hline AtGRF4 & At3g52910 & - & - & - & & - & - \\
\hline AtGRF5 & At3g13960 & - & - & BrGRF5 & Bra027384 & 1164 & 387 \\
\hline AtGRF6 & At2g06200 & - & - & - & & - & - \\
\hline AtGRF7 & At5g53660 & $\mathrm{KBrB} 078 \mathrm{H} 21$ & AC189470 & $B r G R F 7$ & Bra022667 & 1047 & 348 \\
\hline \multirow[t]{2}{*}{$A t G R F 8$} & At4g24150 & KBrH123I10 & AC241192 & $B r G R F 8-1$ & Bra019244 & 1260 & 419 \\
\hline & & KBrH003F06 & AC172868 & $B r G R F 8-2$ & Bra013767 & 1209 & 402 \\
\hline AtGRF9 & At2g45480 & KBrB073H13 & AC232521 & $B r G R F 9$ & Bra039334 & 1317 & 438 \\
\hline
\end{tabular}

${ }^{\mathrm{a}} \mathrm{BAC}$ clones were selected from the BrGP database. 


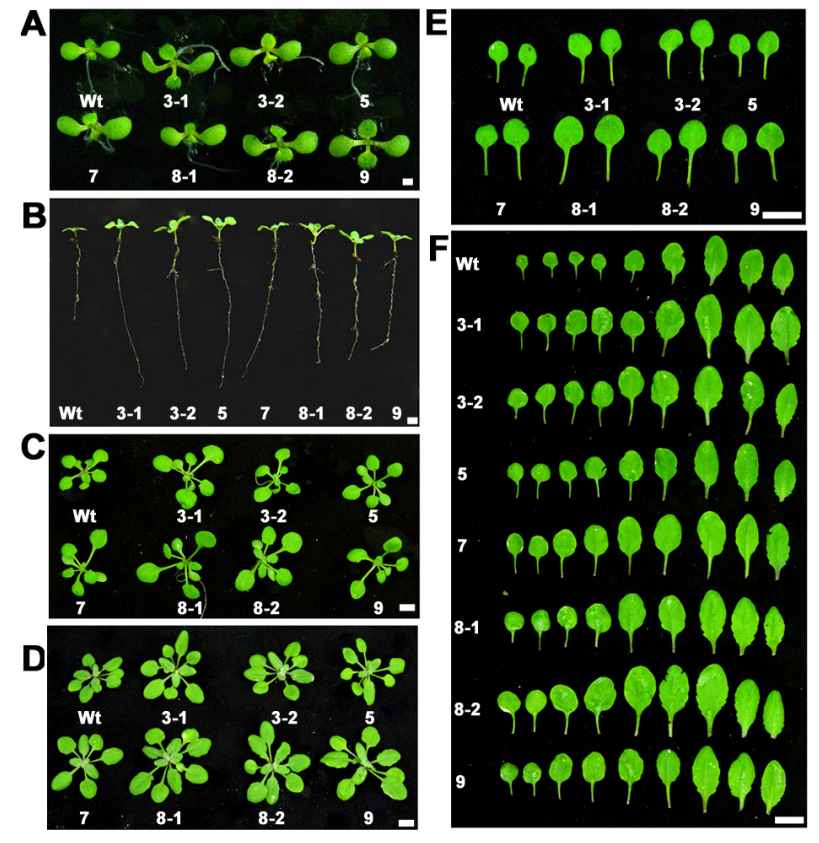

Fig. 1 Phenotypes of $B r G R F-O X$ plants. (A) Eight-day-old $B r G R F$ OX seedlings. The bar indicates $1 \mathrm{~mm}$. (B) Primary root elongation. Bar, $1 \mathrm{~mm}$. (C) and (D) Soil-grown plants at 15 and 20 days, respectively. Bar, $1 \mathrm{~cm}$. (E) and (F) Leaf size and morphology at 15 and 22 days, respectively. Bar, $1 \mathrm{~cm}$. Wt, untransformed wildtype plants; the numbers indicate line numbers of $B r G R F-\mathrm{OX}$

cDNA and BAC clone sequences indicated that all BrGRF proteins consisted of 348 to 518 amino acid residues and contained the conserved QLQ and WRC domains in the $\mathrm{N}$-terminal region (Table 1; Wang et al. 2014). Seven BrGRFs (BrGRF3-1, 3-2, 5, 7, 8-1, 8-2, and 9) under the control of the CaMV $35 \mathrm{~S}$ promoter were introduced into $A$. thaliana plants by $A$. tumefaciens-mediated transformation. We selected 30 independent $T_{1}$ plants on selection media containing hygromycin or phosphinothricin. Genomic PCR analyses confirmed the presence of $B r G R F$ in each line (data not shown). Transgenic lines displaying the typical 3-to-1 segregation ratio were selected, and thus all 21 lines for seven BrGRFs (3 lines per each gene) were established to contain a single T-DNA insertion (Supplemental Fig. 1B). RT-PCR analysis of $\mathrm{T}_{3}$ lines confirmed that the established transgenic plants overexpress $B r G R F \mathrm{~s}$, thus named $B r G R F$-OX (Supplemental Fig. 1C).

Pleiotropic phenotypes of $B r G R F$-OX plants with respect to growth and development

We analyzed dimensional parameters of cotyledons and leaves of all $B r G R F-O X$ plants at different developmental stages. Cotyledons of 8-day-old $B r G R F$-OX seedlings were larger than those of the wild type (Fig. 1A). Quantitative measurements revealed that the surface area and petiole length of $B r G R F-O X$ cotyledons increased by 32 to $51 \%$ and 55 to $100 \%$, respectively, over those of the wild type (Fig. 2A). $B r G R F-O X$ plants also had longer primary roots compared with the wild type at the same stage (Fig. 1B, 2E).

The leaf sizes of $B r G R F-O X$ lines were also larger than those of the wild type throughout the vegetative growth stages (Fig. 1C-F). The second leaves of 15-day-old plants and the fourth leaves of 20-day-old plants were used for the determination of surface areas. As a result, surface areas of 15-day-old and 20-day-old BrGRF-OX leaves increased by 18 to $130 \%$ and 56 to $69 \%$, respectively, compared with those of the wild type (Fig. 2B, 2C). The increases in the surface area resulted from increases in both length and width of leaf blades. Petiole lengths of BrGRF-OX leaves also increased. These results are similar to those of AtGRF-OX plants (Horiguchi et al. 2005; Kim et al. 2003; Wang et al. 2014). Bolting and flowering times of $B r G R F$ OX plants were not different from those of the wild type (data not shown). BrGRF-OX plants also had larger flowers than the wild type, developing normal stamens and gynoecia (Fig. 2D, 3A). The surface area of BrGRF-OX petals increased by 13 to $30 \%$ over that of the wild type (Fig. 2D). The increases in the surface area of petals were due to increases in both length and width. These results demonstrate that $B r G R F$ overexpression increases plant organ size: influences of BrGRF8-1-OX and BrGRF8-2-OX are greater than those of the others. Wang et al. (2014) also reported similar data, in which $B r G R F 8$ overexpression in A. thaliana increased sizes of leaves and other organs by regulating cell proliferation. Interestingly, $B r G R F-\mathrm{OX}$ plants had larger siliques and seeds than the wild type (Fig. 3B, 3C). Morphological observation revealed that both the length and width of seeds increased significantly in BrGRF-OX lines compared with the wild type (Table 2), but seed number per silique of transgenic plants was similar to the wild type (data not shown). Weight and oil contents of BrGRF-OX seeds increased by 14 to $34 \%$ and 15 to $61 \%$, respectively, compared with wild-type seeds. In the transgenic plant seeds, the amount of most fatty acids increased compared with the wild-type, however the composition of various fatty acids in the transgenic plant seeds are not significantly changed compared with the wild-type (Supplemental Table 5). Similarly, it has been reported that up-regulation of OsGRF4 in rice and overexpression of $B n G R F 2$ in A. thaliana greatly enhanced the size, weight, and yield of seeds (Che et al. 2016; Duan et al. 2016; $\mathrm{Hu}$ et al. 2015; Li et al. 2016; Liu et al. 2012). In addition, Arabidopsis seeds overexpressing $B n G R F 2$ contained $10 \%$ more oil than wild- type seeds (Liu et al. 2012). 

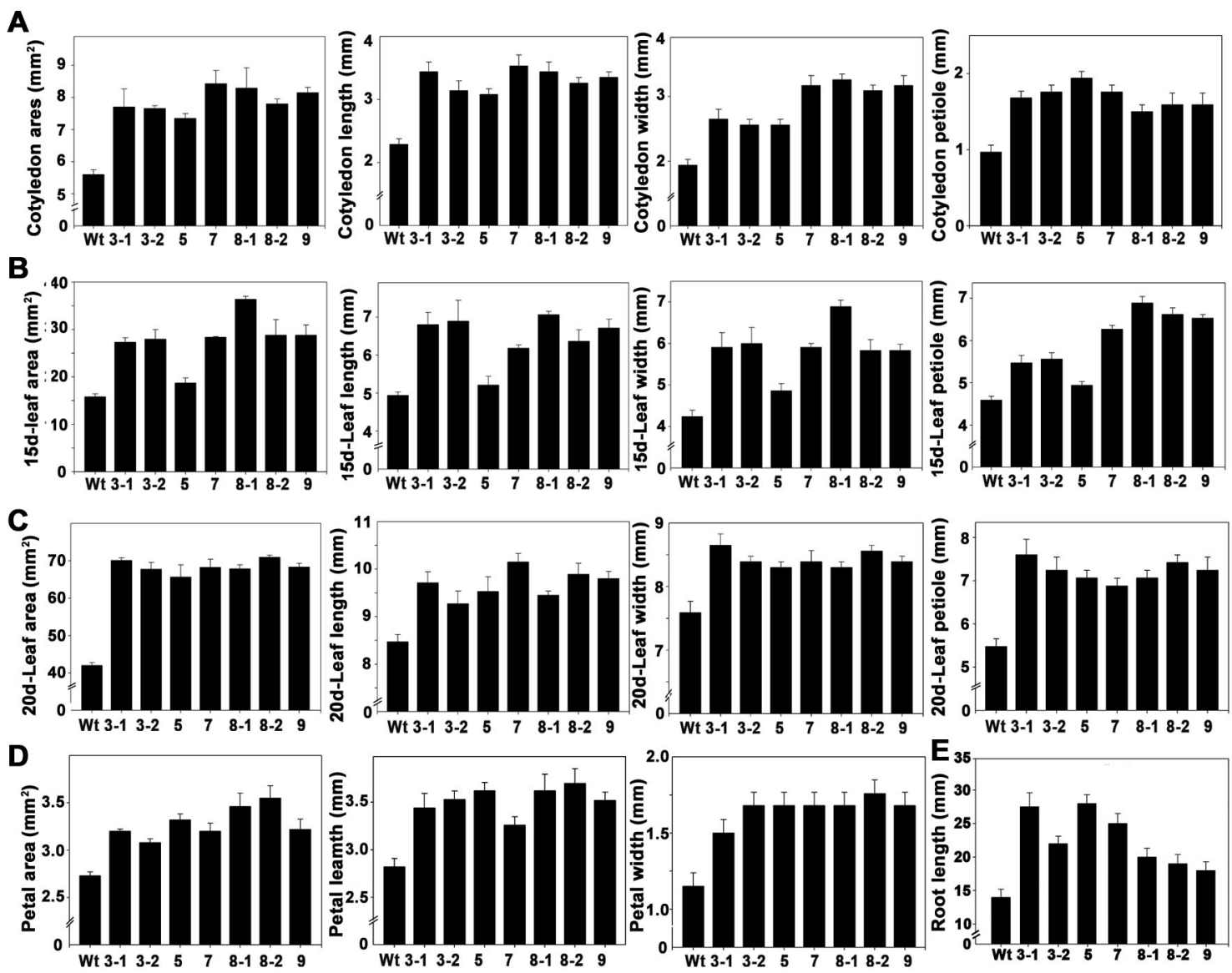

Fig. 2 Quantification of dimensional parameters of BrGRF-OX organs. (A) Cotyledons at 8 days. (B) and (C) The 2nd and 4th leaves at 15 and 20 days, respectively. (D) Mature petals at 35 days. (E) Length of primary roots of 8-day-old seedlings. Error bars represent the standard error (SE). Wt, untransformed wild-type plants; the numbers at $\mathrm{X}$ axes indicate line numbers of $B r G R F$-OX

Table 2 Seed characters of $B r G R F-O X$ plants

\begin{tabular}{lcccc}
\hline \multicolumn{1}{c}{ Transgenic Plant } & Seed length $(\mathrm{mm})$ & Seed width $(\mathrm{mm})$ & $\begin{array}{c}\text { Seed weight } \\
(\mathrm{mg} / 100 \text { seeds })\end{array}$ & $\begin{array}{c}\text { Seed lipids } \\
(\mathrm{mg} / 100 \text { seeds })\end{array}$ \\
\hline Wt & $0.465 \pm 0.003$ & $0.265 \pm 0.002$ & $1.64 \pm 0.02$ & $0.66 \pm 0.070$ \\
BrGRF3-1 & $0.552 \pm 0.002$ & $0.308 \pm 0.003$ & $2.01 \pm 0.01$ & $0.87 \pm 0.078$ \\
BrGRF3-2 & $0.518 \pm 0.003$ & $0.313 \pm 0.003$ & $2.14 \pm 0.02$ & $1.06 \pm 0.073$ \\
BrGRF5 & $0.553 \pm 0.002$ & $0.321 \pm 0.002$ & $1.87 \pm 0.02$ & $0.76 \pm 0.049$ \\
BrGRF7 & $0.547 \pm 0.003$ & $0.313 \pm 0.003$ & $2.19 \pm 0.01$ & $0.98 \pm 0.054$ \\
BrGRF8-1 & $0.522 \pm 0.003$ & $0.314 \pm 0.002$ & $2.06 \pm 0.02$ & $1.03 \pm 0.084$ \\
BrGRF8-2 & $0.567 \pm 0.002$ & $0.318 \pm 0.003$ & $2.20 \pm 0.05$ & $1.02 \pm 0.016$ \\
BrGRF9 & $0.528 \pm 0.003$ & $0.314 \pm 0.003$ & $2.09 \pm 0.02$ & $1.00 \pm 0.074$ \\
\hline
\end{tabular}

Overexpression of BrGRFs enhances organ growth by promoting cell proliferation

The final size of plant organs is a result of the coordinated action of two processes, i.e., cell division and cell expansion (Gonzalez et al 2010). To examine the cellular basis for the alterations in organ size, we observed adaxial palisade cells of cotyledons and leaves as wells as epidermal cells of petals, and found that there was no significant differences in cell sizes between the BrGRF-OX and the wild-type organs (Fig. 4). This observation was confirmed by quantitative analysis as well (Fig. 5). In contrast, total numbers of cells in cotyledons, leaves, and petals of $B r G R F-O X$ plants signi ficantly increased by 33.7 to $55.8 \%, 15$ to $130 \%$, and 19 to $45.1 \%$, respectively, in comparison with the wild type (Fig. 5). These results indicate therefore that the large size 


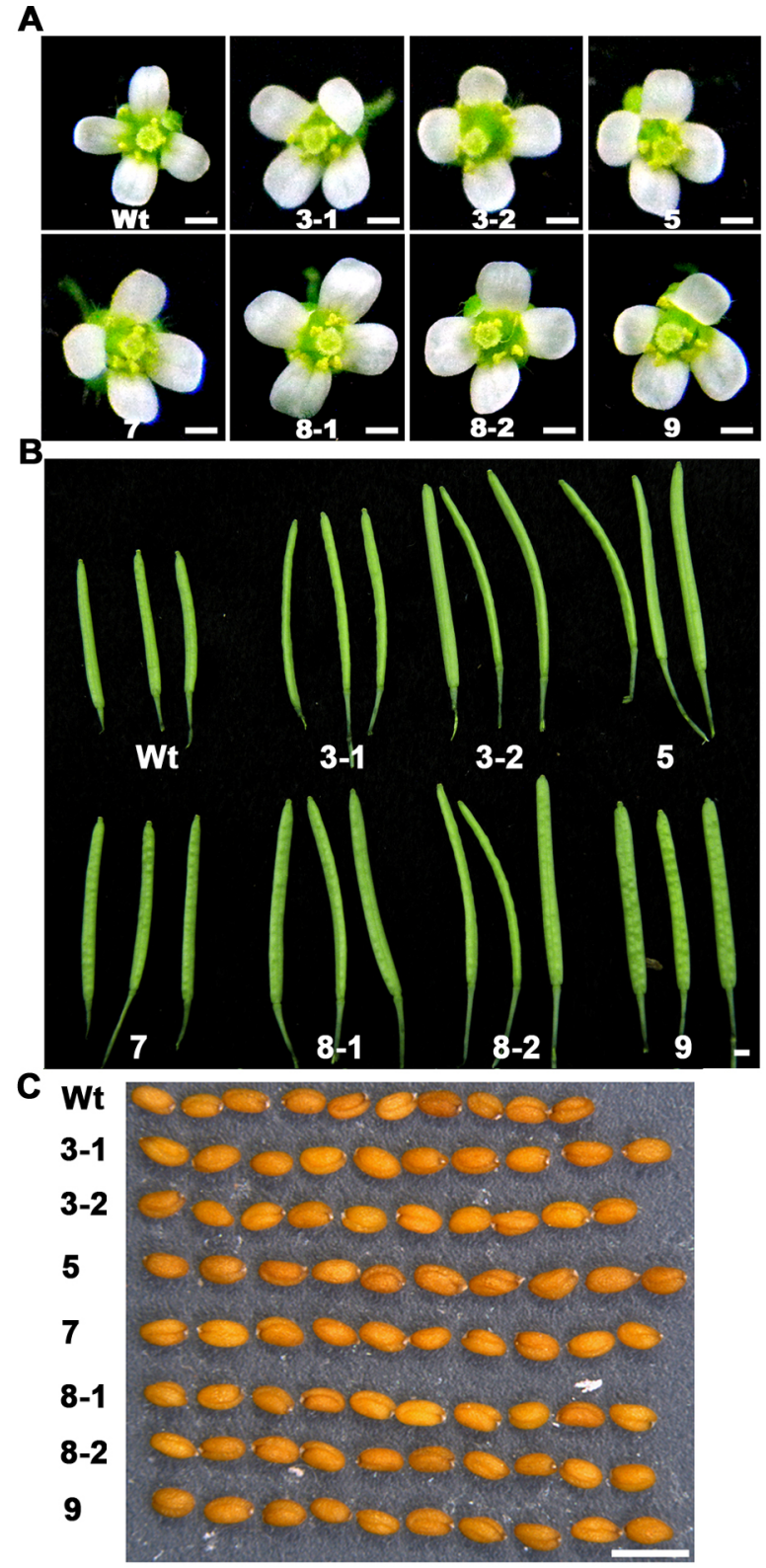

Fig. 3 Phenotypic characters of reproductive organs and seeds of BrGRF-OX. (A) Flowers, (B) Siliques, and (C) Seeds. Wt, untransformed wild-type plants; the numbers indicate line numbers of BrGRF-OX. Bars, $1 \mathrm{~mm}$

of $B r G R F-O X$ organs is due to increases in cell numbers, but not due to increases in cell size. Our data are well in agreement with previous studies in which overexpression of $A t G R F s, B n G R F 2$, and $B r G R F 8$ promoted cell proliferation activities and thus increased organ size (Kim and Lee 2006; Liu et al. 2012; Wang et al. 2014). In conclusion, the seven $B r G R F s$ examined in this study seem to act as positive regulators of the cell proliferation process, consequently determining the final size of organs.

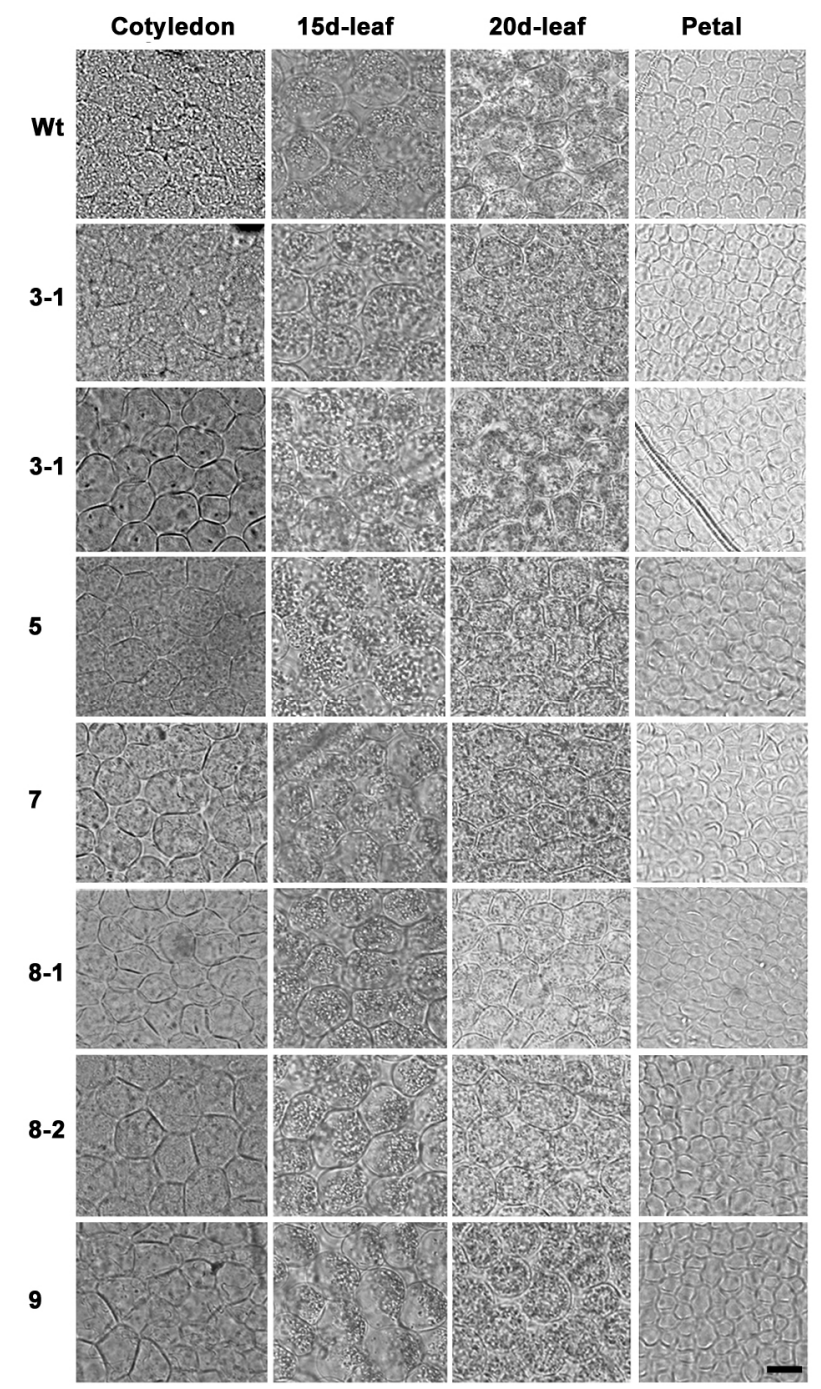

Fig. 4 Microscopic views of adaxial palisade cells in cotyledons and leaves, and epidermal cells of petals of BrGRF-OX. Wt, untransformed wild-type plants; the numbers indicate line numbers of BrGRF-OX. Bar, $10 \mu \mathrm{m}$

Expression patterns of cell division- and hormone-related genes in $B r G R F$-OX plants

Since $B r G R F-O X$ lines resulted in increases in cell number and organ size, it is conceivable that $B r G R F s$ may regulate the expression of GA-, auxin- and cell division-related genes involved in the control of organ growth. The notion prompted us to analyze expression patterns of 15 GA-, 12 auxin-, and 39 cell division/expansion-related genes using semi-quantitative RT-PCR in shoot apical tissues and leaves. In shoot apical tissues, expression levels of GA-related $G L 1$, auxin-related $A X R 1$, and cell division-related $A T H B 16$ were increased by $B r G R F$ overexpression, whereas those of cell expansion-related genes, $E B P 1$ and $E X P 3$, were reduced (Fig. 6A). It has been shown that $A T H B 16$ functions as a negative regulator of leaf 


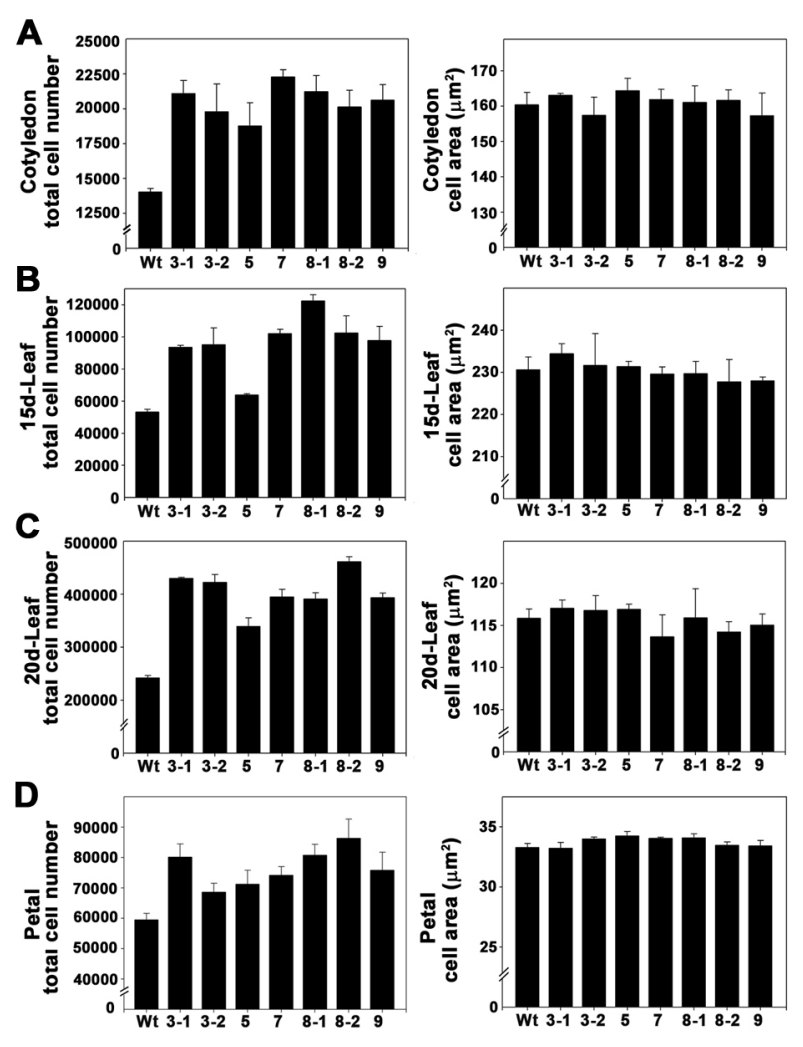

Fig. 5 Numbers and sizes of adaxial palisade cells in cotyledons and leaves, and epidermal cells of petals of BrGRF-OX. (A) Cotyledons at 8 days. (B) and (C) The 2 nd and 4 th leaves at 15 and 20 days, respectively. (D) Mature petals at 35 days. Error bars represent the standard error (SE). Wt, untransformed wild-type plants; the numbers at $\mathrm{X}$ axes indicate line numbers of $B r G R F-\mathrm{OX}$

cell expansion (Wang et al. 2003); that the ectopic expression of cell expansion-related EXP10, EXP3, and EBP1 genes leads to larger leaves and longer petioles by enlarging cell size (Cho and Cosgrove 2000; Horváth et al. 2006; Gonzalez et al. 2009; Kwon et al. 2008). Therefore, our results suggest that, in shoot apical tissues, BrGRFs may up-regulate GA-, auxin-, and cell division-related genes, thus promoting organ growth via enhancement of cell division, whereas they may act to restrict expression of cell expansion-related genes. In $B r G R F-O X$ leaves, expression levels of GA-related GAMYB65/SOC, auxin-related IAMT1/IAA3/IAA6/IAA16/SUR1/ SUR2/AXR1, and cell division-related CYCB1;1/CYCB 2;1/ $D W F 4 / E X O R / A V P 1 / D A 1$ all increased, although their expression levels varied depending on $B r G R F-\mathrm{OX}$ lines (Fig. 6B). Previous studies have shown that GA- and auxin-related genes involved in their biosynthesis, transport, or signaling responses play critical roles in organ morphogenesis (Fleet and Sun 2005; $\mathrm{Li}$ et al. 2007). For example, overexpression of GA-related genes resulted in increases in leaf lobe formation and plant growth (Fleet and Sun 2005). The auxin-related genes exerted

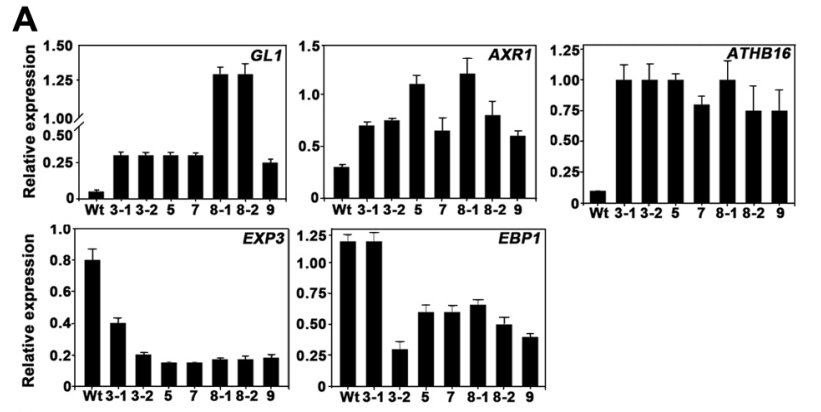

B

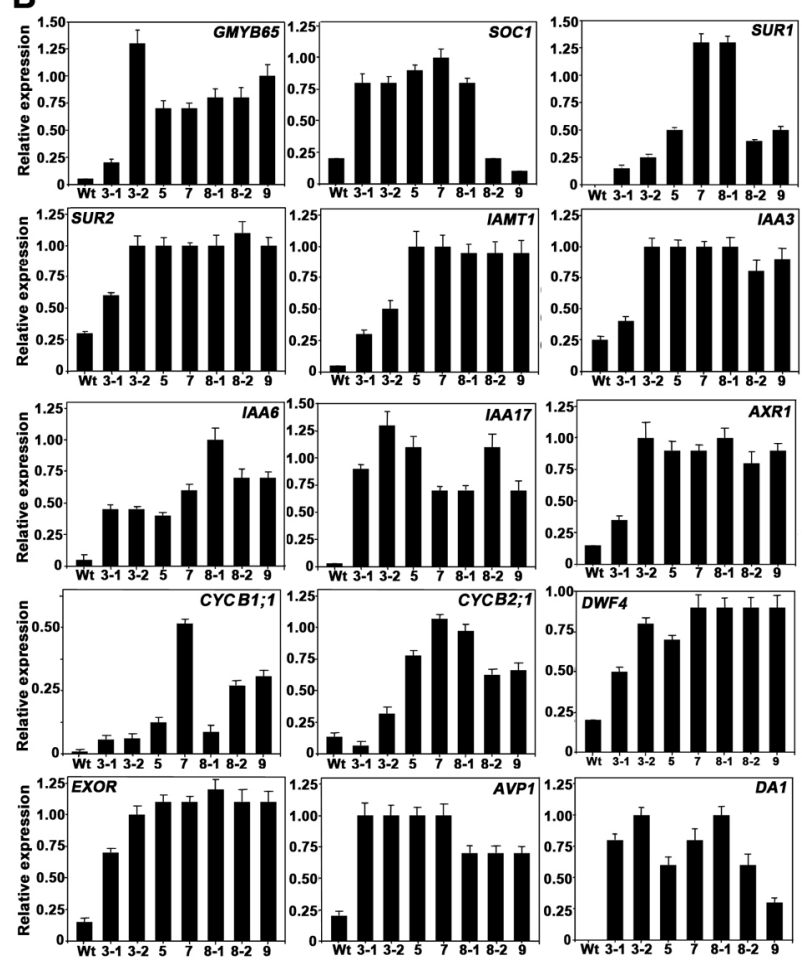

Fig. 6 Expression patterns of GA-, auxin-, and cell division-related genes. (A) Shoot apical tissues. (B) Leaves. Error bars represent the standard error (SE). Wt, untransformed wild-type plants; the numbers at $\mathrm{X}$ axes indicate line numbers of BrGRF-OX. ATHB17, ARABIDOPSIS THALIANA HOMEOBOX-LEUCINE ZIPPER PROTEIN 17; AVP1, PYROPHOSPHATE-ENERGIZED VACUOLAR MEMBRANE PROTON PUMP 1; AXR1, AUXIN RESISTANT 1; CYCB1;1, CYCLIN-B1-1; CYCB2;1, CYCLIN-B2-1; DA1, Protein DA1; DWF4, DWARF 4; EBP1, ERBB-3 BINDING PROTEIN 1; EXOR, EXORDIUM, EXP3, EXPANSIN3; GL1, GLABRA 1; GMYB65, MYB DOMAIN PROTEIN 65; IAA3, 6, and 17, INDOLE-3-ACETIC ACID INDUCIBLE 3, 6, and 17; IAMT1, IAA CARBOXYLMETHYLTRANSFERASE 1; SUR1 and 2, SUPERROOT 1 and 2

major effects on plant growth and development by regulating cell division and cell expansion (Li et al. 2007; PerrotRechenmann 2010). The fact that the expression of $C Y C B 1 ; 1$ and $C Y C B 2 ; 1$ was increased by $B r G R F$ overexpression indicates that $B r G R F$ s may control the cell proliferation activities by positively regulating expression of cell cycle genes in leaves, as described by Lee et al. (2009). It has been shown that the overexpression of DWF4 and EXOR 
results in larger leaves with longer petioles and enhances organ growth (Choe et al 2001; Coll-Garcia et al. 2004). $A V P 1$ is involved in the control of auxin transport, and its overexpression dramatically enhanced organ growth by increasing cell number ( $\mathrm{Li}$ et al. 2005). DAl also controls the final size of seeds and organs in $A$. thaliana by regulating the period of cell proliferation ( $\mathrm{Li}$ et al. 2008). Taken together, we suggest that transcriptional regulation of those genes by $B r G R F-O X$ may result in bigger organ size by increasing cell numbers.

\section{Conclusion}

We have investigated the biological function of seven $B r G R F s$ by overexpressing them in A. thaliana, and found that $B r G R F-O X$ plants produced larger cotyledons, leaves, flowers, and seeds. The results indicate that $B r G R F \mathrm{~s}$ act as positive regulators in regulation of growth and development of plant organs. The positive effect resulted from enhancement of cell proliferation activities. We also found that $B r G R F$ s regulate expression of several GA-, auxin-, and cell division-related genes involved in the control of organ growth. Therefore, we propose that $B r G R F s$ can be utilized for improvement of agronomically important traits of crop plants.

\section{Acknowledgments}

This work was carried out with the support of "Research Program for Agricultural Biotechnology (PJ011813)", National Institute of Agricultural Science, Rural Development Administration, Republic of Korea. J. K. Hong was supported by a 2017 Post-Doctoral Fellowship Program of National Institute of Agricultural Science, Rural Development Administration, Republic of Korea.

\section{References}

AOCS (1997) Fatty acid composition by gas chromatography. In Official Methods and Recommended Practices of the American Oil Chemists. Method Ce 1-62 Champaign IL USA

Cho H-T, Cosgrove DJ (2000) Altered expression of expansin modulates leaf growth and pedicel abscission in Arabidopsis thaliana. Proc Natl Acad Sci USA 97:9783-9788

Choe S, Fujioka S, Noguchi T, Takatsuto S, Yoshida S, Feldmann KA (2001) Overexpression of DWARF4 in the brassinosteroid biosynthetic pathway results in increased vegetative growth and seed yield in Arabidopsis. Plant J 26:573-582

Coll-Garcia D, Mazuch J, Altmann T, Müssig C (2004) EXORDIUM regulates brassinosteroid-responsive genes. FEBS Lett 563:82-86

Che R, Tong H, Shi B, Liu Y, Fang S, Liu D, Xiao Y, Hu B, Liu L, Wang H, Zhao M, Chu C (2016) Control of grain size and rice yield by GL2-mediated brassinosteroid responses. Nature Plants 2:15195

Desfeux C, Clough SJ, Bent AF (2000) Female reproductive tissues are the primary target of Agrobacterium-mediated transformation by the Arabidopsis floral-dip method. Plant Physiol 123:895-904

Duan P, Ni S, Wang J, Zhang B, Xu R, Wang Y, Chen H, Zhu X, Li Y (2016) Regulation of OsGRF4 by OsmiR396 controls grain size and yield in rice. Nature Plants 2:15203

Fleet CM, Sun TP (2005) A DELLAcate balance: the role of gibberellins in plant morphogenesis. Curr Opin Plant Biol 8:77-85

Gonzalez N, De Bodt S, Sulpice R, Jikumaru Y, Chae E, Dhondt S, Van Daele T, De Milde L, Weigel D, Kamiya Y, Stitt M, Beemster GT, Inzé D (2010) Increased leaf size: different means to an end. Plant Physiol 153:1261-1279

Gonzalez N, Beemster G, Inzé D (2009) David and Goliath: what can the tiny weed Arabidopsis teach us to improve biomass production in crops?. Curr Opin Plant Biol 12:157-164

Horváth BM, Magyar Z, Zhang Y, Hamburger AW, BakóL, Visser RGF, Bachem CWB, Bögre L (2006) EBP1 regulates organ size through cell growth and proliferation in plants. EMBO J 25:4909-4920

Horiguchi G, Kim GT, Tsukaya H. (2005) The transcription factor AtGRF5 and the transcription coactivator AN3 regulate cell proliferation in leaf primordia of Arabidopsis thaliana. Plant J 43:68-78

Hu J, Wang Y, Fang Y, Zeng L, Xu J, Yu H, Shi Z, Pan J, Zhang D, Kang S, Zhu L, Dong G, Guo L, Zeng D, Zhang G, Xie L, Xiong G, Li J, Qian Q (2015) A rare allele of GS2 enhances grain size and grain yield in rice. 8:1455-1465

Kim JH, Choi D, Kende H (2003) The AtGRF family of putative transcription factors is involved in leaf and cotyledon growth in Arabidopsis. Plant J 36:94-104

Kim JH, Kende H (2004) A transcriptional coactivator, AtGIF1, is involved in regulating leaf growth and morphology in Arabidopsis. Proc Natl Acad Sci USA 101:13374-13379

Kim JH, Lee BH (2006) GROWTH-REGULATING FACTOR4 of Arabidopsis thaliana is required for development of leaves, cotyledons, and shoot apical meristem. J Plant Biol 49:463-468

Kim JH, Tsukaya H (2015) Regulation of plant growth and development by the GROWTH-REGULATING FACTOR and GRF-INTERACTING FACTOR duo. J Exp Bot 66: 6093-6107

Krizek BA (2009) Making bigger plants: key regulators of final organ size. Curr Opin Plant Biol 12:17-22

Kwon YR, Lee HJ, Kim KH, Hong S-W, Lee SJ, Lee H (2008) Ectopic expression of Expansin3 or Expansin $\beta 1$ causes enhanced hormone and salt stress sensitivity in Arabidopsis. Biotech Lett 30:1281-1288 
Lee BH, Ko J-H, Lee S, Lee Y, Pak J-H, Kim JH (2009) The Arabidopsis GRF-INTERACTING FACTOR gene family performs an overlapping function in determining organ size as well as multiple developmental properties. Plant Physiol 151:655-668

Li J, Yang H, Peer WA, Richter G, Blakeslee J, Bandyopadhyay A, Titapiwantakun B, Undurraga S, Khodakovskaya M, Richards EL (2005) Arabidopsis $\mathrm{H}^{+}$-PPase AVP1 regulates auxin-mediated organ development. Science 310:121-125

Li LC, Kang DM, Chen ZL, Qu LJ 2007. Hormonal regulation of leaf morphogenesis in Arabidopsis. J Integr Plant Biol 49:75-80

Li S, Gao F, Xie K, Zeng X, Cao Y, Zeng J, He Z, Ren Y, Li W, Deng Q, Wang S, Zheng A, Zhu J, Liu H, Wang L, Ping Li (2016) The OsmiR396c-OsGRF4-OsGIF1 regulatory module determines grain size and yield in rice. Plant Biotech $\mathrm{J}$ 14:2134-2145

Li Y, Zheng L, Corke F, Smith C, Bevan MW (2008) Control of fi nal seed and organ size by the DA1 gene family in Arabidopsis thaliana. Genes Dev 22:1331-1336

Liang G, He H, Li Y, Wang F, Yu D (2014) Molecular mechanism of microRNA396 mediating pistil development in Arabidopsis. Plant Physiol 164:249-258

Liang YC, Jeon Y-A, Lim S-H, Kim JK, Lee J-Y, Kim Y-M, Lee Y-H, Ha S-H(2011) Vascular-specific activity of the Arabidopsis carotenoid cleavage dioxygenase 7 gene promoter. Plant Cell Rep 30:973-980

Liu D, Song Y, Chen Z, Yu D (2009) Ectopic expression of miR396 suppresses GRF target gene expression and alters leaf growth in Arabidopsis. Physiol Plantarum 136:223-236

Liu J, Hua W, Yang HL, Zhan GM, Li RJ, Deng LB, Wang XF, Liu GH, Wang HZ (2012) The BnGRF2 gene (GRF2-like gene from Brassica napus) enhances seed oil production through regulating cell number and plant photosynthesis. J Exp Bot 63:3727-3740

Murashige T, Skoog F (1962) A revised medium for rapid growth and bioassays with tobacco tissue cultures. Physiol Plantarum 15:473-497

Omidbakhshfard MA, Proost S, Fujikura U, Mueller-Roeber B (2015) Growth-Regulating Factors (GRFs): a small transcription factor family with important functions in plant biology. Mol Plant 8:998-1010

Perrot-Rechenmann C (2010) Cellular responses to auxin: division versus expansion, Cold Spring Harb Perspect Biol 2:a001446

Van der Knaap E, Kim JH, Kende H (2000) A novel gibberellin induced gene from rice and its potential regulatory role in stem growth. Plant Physiol 122:695-704

Wang F, Qiu N, Ding Q, Li J, Zhang Y, Li H, Gao J (2014) Genome-wide identification and analysis of the growth-regulating factor family in Chinese cabbage (Brassica rapa L. ssp. pekinensis). BMC Genomics 15:807

Wang Y, Henriksson E, Söderman E, Henriksson KN, Sundberg E, EngströmP (2003) The Arabidopsis homeobox gene, ATHB16, regulates leaf development and the sensitivity to photoperiod in Arabidopsis. Dev Biol 264:228-239

Ye R, Yao QH, Xu ZH, Xue HW (2004) Development of an efficient method for the isolation of factors involved in gene transcription during rice embryo development. Plant J 38: 348-357

Zhang DF, Li B, Jia GQ, Zhang TF, Dai JR, Li JS, Wang SC (2008) Isolation and characterization of genes encoding GRF transcription factors and GIF transcriptional coactivators in maize (Zea mays L.). Plant Sci 175:809-817 